\title{
Rotational velocity distributions of A-type stars
}

\author{
F. Royer ${ }^{1,2}$, J. Zorec ${ }^{3}$ and A.E. Gómez ${ }^{2}$ \\ ${ }^{1}$ Observatoire de Genève, 51 chemin des maillettes, 1290 Sauverny, Switzerland \\ email: frederic.royer@obs.unige.ch \\ ${ }^{2}$ GEPI/CNRS UMR 8111, Observatoire de Paris-Meudon, \\ 92195 Meudon cedex, France \\ ${ }^{3}$ Institut d'Astrophysique de Paris, 98bis boulevard Arago, 75014 Paris, France
}

\begin{abstract}
Using an homogeneous sample of $v \sin i$ values for A-type main sequence stars (Royer et al. 2002), the equatorial velocity $(v)$ distributions are determined as function of spectral class, from B9 to F2. The chemically peculiar and binary stars are discarded. These distributions of "normal" stars are discussed in terms of stellar formation and evolution, in particular the remaining bimodality observed for the earliest spectral types of the sample. We show that late B and early A-type main-sequence stars have genuine bimodal distributions of true equatorial rotational velocities probably due to the phenomena of angular momentum loss and of redistribution the star underwent before reaching the main sequence. A striking lack of slow rotators is noticed among intermediate and late A-type stars. The bimodal-like shape of their true equatorial rotational velocity distributions could be due to evolutionary effects.
\end{abstract}

Keywords. Stars: early-type, stars: rotation

\section{Introduction}

Thirty years ago investigations by Deutsch (1970) and Dworetsky (1974) already focused on the rotational velocity distributions of A-type stars and the bimodality observed around A0 in particular. Guthrie (1982) found that cluster late B-type stars have bimodal rotational velocity distributions, while for the same class of field stars they are unimodal. Bimodality was also observed in rotation of solar mass stars in Orion by Attridge \& Herbst (1992), Choi \& Herbst (1996) and Herbst et al. (2001).

Thus since bimodal distributions were found for cluster late B-type stars and for solar mass stars, it is important to review the distributions of rotational velocities of field late B-type stars in the early MS phases, to see whether it is possible to detect signatures of bimodality using new and highly homogeneous data. In the same way, it is also significant to know whether distributions of rotational velocities of stars in the spectral range from A0 to F0 are bimodal. This spectral range is of particular interest because it represents the transition from objects with radiative to those with convective envelopes.

Apart from the distinct type of stellar formation evoked by Guthrie (1982) to account for the velocity distribution aspects, fragmentation of large clouds for the bimodal star sample and through turbulence in small clouds in loose clusters for the unimodally distribution, differences could be also searched in circumstances that could lead in each case to a distinct internal angular momentum redistribution. A higher concentration of the angular momentum towards the stellar core might trigger phenomena with surface proxies noted as "stellar peculiarities". In this context can be considered the study of Abt \& Morrell (1995) who tried to associate the Am phenomenon to the slow-rotation excess in the velocity distributions of A-type stars. 
Thus, according to Abt \& Morrell's (1995) attempt and the comments above, the goals of the paper are a) to see whether the chemically peculiar characteristics and the excess of slow rotators among the more massive stars of our sample, late type B and early A-type stars, are necessarily concomitant phenomena and b) to investigate whether the rotational velocity distributions of intermediate and late A-type stars bear signatures that could be related to the complex structure of their envelopes.

The full study will be published in a forthcoming paper (Royer et al. 2004).

\section{Rotational velocities data}

This study is based on the catalog of Royer et al. (2002), which compiles homogeneous $v \sin i$ data for $\mathrm{B} 8$ to $\mathrm{F} 2$-type stars. The luminosity class range $\mathrm{V}$ to $\mathrm{IV}$ is used as synonymous for "dwarfs" representing the first MS evolutionary phases. The first selection is then based on the luminosity class of the stars, and only the main sequence stars (classes $\mathrm{V}, \mathrm{IV}-\mathrm{V}$ and IV) are retained.

In this study, all known "close" binaries and chemical peculiar stars in the studied spectral range have been removed. Binaries were eliminated because of possible tidal braking mechanisms which introduce deviations in the distributions that cannot be ascribed to initial MS conditions related to single stars. Following the same line of thought, Am stars were taken out of the studied sample, as they are considered to be close binaries (Debernardi 2000). Moreover, they are found among the slow rotators (Abt \& Morrell 1995).

Evidence exists that the Ap phenomenon appears when the star has already completed at least the first third of its MS life span (Hubrig et al. 2000a). Ap stars represent then a population which is systematically separated from what it could be meant as the first MS stages. No indications were found for significant magnetic braking in the stellar surface (Hubrig et al. 2000b). Be and A-type stars with shell spectra were also removed. A-shell stars seem to be the A-type counterparts of early-type Be stars (Abt \& Moyd 1973). The Be phenomenon is associated with fast rotation and is age-dependent (Zorec 2004).

CP stars (chemically peculiar stars): The catalogs of Ap and Am stars from Renson et al. (1991) are used to identify peculiar stars, as well as the spectral classifications of Abt \& Morrell (1995) and Abt et al. (2002).

CB stars ("close" binary stars): Tidal effects in a multiple system tend to synchronize the axial rotation period with the orbital period. This category of stars are selected using criteria based on HIPPARCOS data and spectroscopic data. Except for a few, the selected stars are in the HIPPARCOS catalog (ESA 1997). The binaries detected by this satellite, with $\Delta H p<4$ mag are flagged as CB stars. The compilation by Pedoussaut et al. (1985) of spectroscopic binaries is used to complete the identification, together with the Eighth Catalog of the Orbital Elements of Spectroscopic Binaries (Batten et al. 1989). Stars with both $\mathrm{CB}$ and $\mathrm{CP}$ criteria are classified as $\mathrm{CP}$.

The sample is divided according to spectral type, chosen so as to warrant in each statistical significance and mass resolution, to allow for the detection of possible mass dependencies of the velocity distributions. The six resulting subsamples are listed in Table 1. Their respective $v \sin i$ distributions are displayed in Fig. 1 as hatched histograms.

\section{Distributions of equatorial rotational velocities}

\subsection{Rectified distributions}

The observed $v \sin i$ parameter is the projection of the equatorial velocity $v$ of the star on the line of sight, $i$ being the inclination between the stellar rotation axis and the line of 


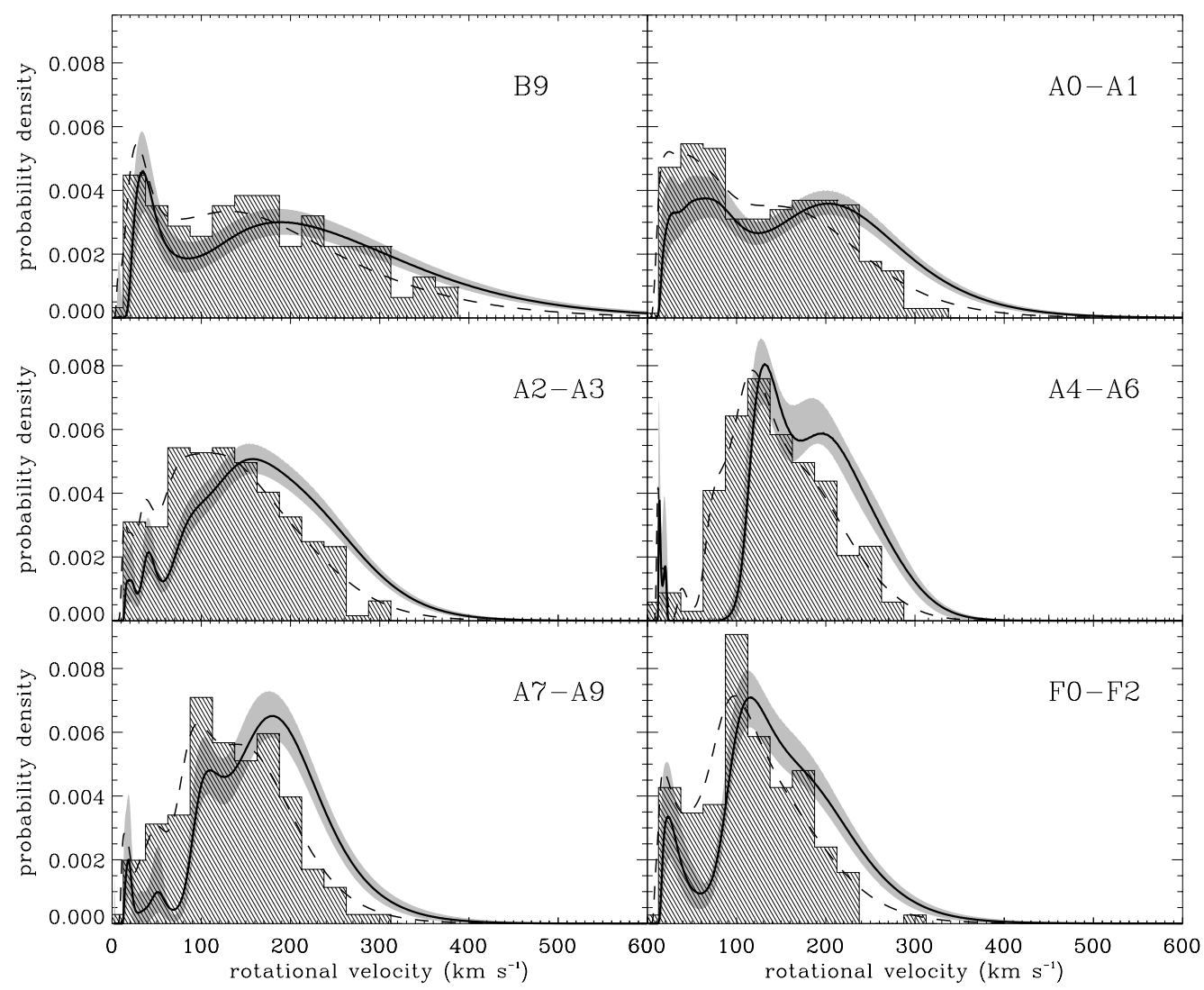

Figure 1. Rotational velocity distributions: observed $v \sin i$ (histograms), fitted $v \sin i$ distributions (dashed lines), deconvolved $v$ distributions (solid lines). The gray strips around the solid lines are the variability bands which shows the significance of the modes in the distribution.

Table 1. Moments of the $v \sin i$ distributions. For each subsample, the number of stars, the median, the mean and the dispersion of the $v \sin i$ distribution are indicated

\begin{tabular}{lccccccc}
\hline Subsample & $\#$ & \multicolumn{3}{c}{$\begin{array}{c}\text { Center } \\
\text { median }\end{array}$} & \multicolumn{2}{c}{$\begin{array}{c}\left.\mathrm{km} \mathrm{s}^{-1}\right) \\
\text { mean }\end{array}$} & \multicolumn{2}{c}{$\begin{array}{c}\text { Disp. } \\
\left(\mathrm{km} \mathrm{s}^{-1}\right)\end{array}$} \\
\hline B9 & 125 & 159 & \pm 11.0 & 161 & \pm 8.7 & 98 & \pm 6.2 \\
A0-A1 & 271 & 128 & \pm 5.9 & 131 & \pm 4.7 & 78 & \pm 3.4 \\
A2-A3 & 258 & 128 & \pm 5.2 & 133 & \pm 4.2 & 67 & \pm 3.0 \\
A4-A6 & 137 & 138 & \pm 6.0 & 144 & \pm 4.8 & 56 & \pm 3.4 \\
A7-A9 & 141 & 133 & \pm 6.3 & 134 & \pm 5.0 & 60 & \pm 3.6 \\
F0-F2 & 150 & 110 & \pm 5.9 & 114 & \pm 4.7 & 57 & \pm 3.3 \\
\hline
\end{tabular}

sight. The assumption that stellar rotation axes are randomly oriented is adopted. This hypothesis has been tested many times (Gray 1992, Gaigé 1993) and is still considered to be valid.

The Probability Density Function (hereafter PDF) of the $v \sin i$ values is thus the result of the convolution between the distribution of "true" equatorial velocities $v$, the distribution of inclination angles $i$, and the observational error law. It is estimated from the observed histograms, using a Kernel method. The smoothed $v \sin i$ distributions are displayed in Fig. 1 (dashed lines). The PDF of $v \sin i$ values is first deconvolved by the 


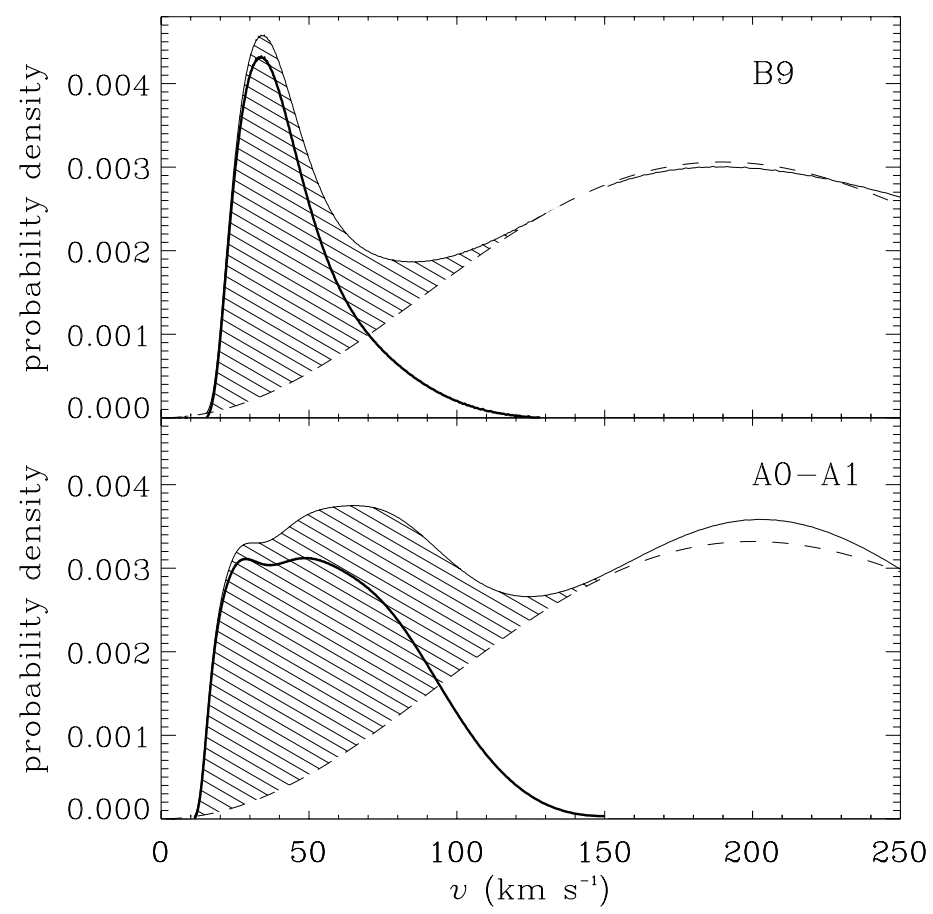

Figure 2. Slow rotators in B9 and A0-A1 stars. The fast rotator modes are fitted by Maxwellian distributions (dashed line). The excesses of slow rotators is represented by the hatched areas. The thick solid lines are the differences between the PDF of the sample and the Maxwellian distribution.

distribution of errors (chosen as log-normal) and then by the distribution of inclinations (axes randomly oriented). Both processes are carried out using the Lucy (1974) iterative method.

The resulting $v$ distributions are displayed for the six subsamples in Fig. 1.

\subsection{Description of the $v$ distributions}

The distributions of true equatorial velocities imply the following :

- The bimodal character of distributions corresponding to B9 and A0-A1 field dwarf stars is clearly present, even for the samples cleaned of all known Am, Ap and close binary stars. For B9-type stars: the mode of slow rotators is $\sim 35 \mathrm{~km} \mathrm{~s}^{-1}$, and for fast rotators $\sim 190 \mathrm{~km} \mathrm{~s}^{-1}$. For the A0-A1 subsample: they are $\sim 60 \mathrm{~km} \mathrm{~s}^{-1}$ and $\sim 200 \mathrm{~km} \mathrm{~s}^{-1}$, respectively. The modes of fast rotators $\left(v \gtrsim 150 \mathrm{~km} \mathrm{~s}^{-1}\right)$, for B9 and A0-A1 type stars, are fitted by Maxwellian PDFs (Fig. 2). The proportion of slow rotators $\left(v \lesssim 150 \mathrm{~km} \mathrm{~s}^{-1}\right)$ is taken as the excess compared to the Maxwellian fast rotator distribution. The slow rotator peak corresponds to some 19 B9-type stars, and about 66 A0-A1-type stars.

The distribution of the rotation velocities of B9 to A1-type "normal" field dwarf stars are genuinely bimodal. This finding is contrary to the results obtained by Guthrie (1982), Abt \& Morrell (1995), and Abt (2000). The presence of modes around 50 and $200 \mathrm{~km} \mathrm{~s}^{-1}$ may be due to formation processes and phenomena of AM loss and redistribution undergone during PMS phases.

- In the A2 to A9 spectral type groups, the small wiggles of the distributions in the velocity interval $0 \lesssim v \lesssim 70 \mathrm{~km} \mathrm{~s}^{-1}$ concern only a negligible fraction of the stars. Moreover the variability bands associated with the distributions argue in the sense that 
the presence of these slow rotators is not significant. These objects are probably unknown synchronized binaries or chemically peculiar stars that pollute the sample of "normal" stars. There is a net lack of rotators with $v \lesssim 70 \mathrm{~km} \mathrm{~s}^{-1}$. An absolute minimum of this fraction is seen in the A4-A6 group.

- An excess of slow rotators with velocities $0 \lesssim v \lesssim 60 \mathrm{~km} \mathrm{~s}^{-1}$ appears in the F0-F2 group. This mode of slow rotators, around $20 \mathrm{~km} \mathrm{~s}^{-1}$, in the distribution for early-F stars, is significant. The $B-V$ distribution for all the "normal" F0-F2 stars, ranging from 0.2 to $0.4 \mathrm{mag}$, shows that all (but one) stars with $v \sin i<75 \mathrm{~km} \mathrm{~s}^{-1}$ have color indices $B-V \gtrsim 0.3$ mag. It is known that the Böhm-Vitense gap occurs at $0.33 \lesssim B-V \lesssim 0.38 \mathrm{mag}$ (D'Antonna et al. 2002) and is a signature of the transition from radiative to convective atmospheres. The group of slow rotators for F0-F2 stars is then probably composed of stars that undergone a braking due to their convective atmosphere.

- The high-velocity side of the distributions of stars with spectral types ranging from A2 to F2 have a double-like structure. A well defined peak can be noticed at $v \simeq 200 \mathrm{~km} \mathrm{~s}^{-1}$ in all groups, except for the F0-F2 stars for which at $v \simeq 200 \mathrm{~km} \mathrm{~s}^{-1}$ there is only an inflection. The maximum of fast rotators in the B9 and A0-A1 groups is also situated at $v=200 \mathrm{~km} \mathrm{~s}^{-1}$, although the corresponding distributions do not show any noticeable double structure.

The double structure might be due to an evolution-fast rotation interplay effect.

\section{References}

Abt, H. A. 2000, ApJ, 544, 933

Abt, H. A., Levato, H. \& Grosso, M. 2002, ApJ, 573, 359

Abt, H. A. \& Morrell, N. I. 1995, ApJS, 99, 135

Abt, H. A. \& Moyd, K. I. 1973, ApJ, 182, 809

Attridge, J. M. \& Herbst, W. 1992, ApJ Lett., 398, L61

Batten, A. H., Fletcher, J. M. \& MacCarthy, D. G. 1989, 8th Catalogue of the orbital elements of spectroscopic binary systems (Victoria: Dominion Astrophysical Observatory)

Choi, P. I. \& Herbst, W. 1996, AJ, 111, 283

D’Antona, F., Montalbán, J., Kupka, F. \& Heiter, U. 2002, ApJ Lett., 564, L93

Debernardi, Y. 2000, in Birth and Evolution of Binary Stars, IAU Symp. 200, ed. B. Reipurth \& H. Zinnecker, 161

Deutsch, A. J. 1970, in Stellar rotation, IAU Colloquium No.4, ed. A. Slettebak, 207

Dworetsky, M. M. 1974, ApJS 256, 101

ESA. 1997, The Hipparcos and Tycho Catalogues, ESA-SP 1200

Gaigé, Y. 1993, A\&̈A, 269, 267

Gray, D. F. 1992, The observation and analysis of stellar photospheres, 2nd edn. (Cambridge University Press)

Guthrie, B. N. G. 1982, MNRAS, 198, 795

Herbst, W., Bailer-Jones, C. A. L., \& Mundt, R. 2001, ApJ Lett., 554, L197

Hubrig, S., North, P. \& Mathys, G. 2000a, ApJ, 539, 352

Hubrig, S., North, P. \& Medici, A. 2000b, A\&A, 359, 306

Lucy, P. 1974, AJ, 79, 745

Pedoussaut, A., Capdeville, A., Ginestet, N. \& Carquillat, J. M. 1985, List of spectroscopic binaries from the Toulouse general catalogue, Observatoire de Toulouse

Renson, P., Gerbaldi, M., \& Catalano, F. A. 1991, A\&SAS, 89, 429

Royer, F., Grenier, S., Baylac, M.-O., Gómez, A. E. \& Zorec, J. 2002, A\&A, 393, 897

Royer, F., Zorec, J. \& Gómez, A. E. 2004, submitted to A $\mathscr{E} A$

Zorec, J. 2004, in Stellar Rotation, IAU Symp. 215, ed. A. Maeder \& P. Eenens, in press 


\section{Discussion}

DWORETSKY: I saw something similar in A0 rotational velocities many years ago (ApJS, 1994). There is a "double-peaked" distribution of $v_{\mathrm{e}}$ even with all known peculiar and binary stars excluded. Helmut Abt saw something similar. Are any of the "genuine" slow rotators actually chemically peculiar, but we do not know it?

ROYER: The proportion of chemically peculiar stars in my sample is fairly constant at $\approx 15 \%$ for stars brighter than $V=6.5$ mag. This magnitude corresponds to the faint limit of the catalogues of Renson et al. (1991) and Abt \& Morell (1995). Making the hypthesis that this prportion remains constant for stars fainter than $V=6.5$, the estimated number of undetected chemically peculiar stars in my sample is about 20 stars, much smaller than the observed excess for slow rotators. 